\title{
Antigen detection in vivo after immunization with different presentation forms of rabies virus antigen, II. Cellular, but not humoral, systemic immune responses against rabies virus immune-stimulating complexes are macrophage dependent
}

\author{
I. J. T. M. CLAASSEN, ${ }^{*}$ A. D. M. E. OSTERHAUS, $†$ M. POELEN $\ddagger$ N. VAN ROOIJEN§ \& E. CLAASSEN*ף * Laboratory \\ for Quality Control, Institute for Animal Science and Health, Lelystad, $\dagger$ Department of Medical Virology and $\mid$ Department of \\ Immunology, Erasmus University, Rotterdam, $\ddagger$ RIVM-LVM, Bilthoven, and §Department of Cell Biology, Free University, \\ Amsterdam, The Netherlands
}

\begin{abstract}
SUMMARY
In this paper we describe the effect of depletion of splenic macrophages on the uptake, and immune response against, different formulations of rabies virus antigen. Splenic macrophages were removed by intravenous injection with clodronate liposomes. $\beta$-propiolacton inactivated rabies virus (RV-BPL) and immune-stimulating complexes (iscom) containing these antigens were given to macrophage-depleted and control mice. In the absence of phagocytic cells in the spleen, antigen is still trapped in the red pulp and to a lesser extent in the peri-arteriolar lymphocyte sheaths (PALS) for both antigen formulations. The localization pattern in the main area of immune response induction, namely the follicles, was unaltered after macrophage depletion. Functionally, the depletion of splenic and liver macrophages had no influence on the induction of specific antibody responses in both RV-BPL or RV-iscom immunized mice, even though the latter presentation form was clearly associated with specific localization in the marginal metallophillic macrophages. In RV-BPL immunized mice, macrophage depletion had no influence on proliferative T-cell responses. However, macrophage-depleted mice that were immunized with RViscom showed a significant decrease in proliferative T-cell responses. These results confirm existing ideas on the spleen as a physical filter rather than an induction site for humoral responses and shed new light on the efficient role of iscoms as antigen-presenting moieties in relation to their specific in vivo localization patterns and partial macrophage dependency.
\end{abstract}

\section{INTRODUCTION}

Several mechanisms have been postulated to explain the relatively high immunogenicity of antigens presented in immunestimulating complexes (iscoms). ${ }^{1,2}$ Their potency can in part be explained by the specific targeting of these structures to antigen-presenting cells (APC) of the immune system. Recently we developed a novel method for the in situ detection of iscoms. ${ }^{3}$ Employing this technique we demonstrated that iscoms containing rabies virus antigen were taken up by a different subset of macrophages in the spleen than inactivated

Received 28 December 1997; revised 1 April 1998; accepted 1 April 1998.

Abbreviations: DiI, 1,1'-dioctadecyl-3,3,3',3'-tetramethylindocarbocyanine perchlorate; iscom, immune stimulating complex; MZM, marginal zone macrophages; MMM, marginal metallophilic macrophages; NSE, non-specific esterase; RPM, red pulp macrophages; RV rabies virus; RV-BPL, $\beta$-propiolacton inactivated rabies virus.

Correspondence: Dr Ivo. J. T. M. Claassen, Laboratory for Quality Control, Institute for Animal Science and Health, PO Box 65, 8200 AB, Lelystad, The Netherlands. rabies virus antigen, thereby possibly explaining the difference in immunogenicity. Furthermore, we found a surprising preference for iscom, rather than intact virus, in the follicular trapping/uptake of these antigens. These results led to a biphasic presentation model for iscom with a specific localization pattern in marginal metallophilic macrophages (MMM), away from antigen-degrading macrophages, in the first phase and direct presentation to follicular cells in the second phase. This model implies an entire or at least partial dependency of the iscom-induced immune response on this particular macrophage subset. From several studies it has now become evident that the spleen processes antigen in two possible ways, depending on different types of non-lymphoid cells. Firstly, there seems to be a macrophage-dependent pathway for particulate-and thymus-independent type 1 (mitogenic polysaccharides) antigens. ${ }^{4}$ Second, there is an important role for the follicular (dendritic and B) cells of the spleen in trapping and presenting soluble protein and TI- 2 antigens to the immune system. ${ }^{5}$ Because both virus (RV-BPL) and iscom cản qualify as particulate antigens it remains to be established whether these antigens also require macrophage (pre)-processing before they can evoke an effective immune response. In view of our 
earlier study on the difference in splenic localization of these two formulations of rabies antigen and based on the available literature suggesting a suppressive rather that immunestimulating role of splenic macrophages we decided to study the contribution of splenic macrophages in these responses. By making use of a well-established technique for splenic macrophage elimination, employing clodronate liposomes, the role of macrophages, as opposed to the function of follicular cells (which are not affected), can be determined. ${ }^{5}$ By making use of the discrete differences in repopulation kinetics of different splenic macrophage subsets ${ }^{6}$ their role in immune response induction could be studied. Furthermore, this elimination model can be combined with the above mentioned labelling technique, thereby enabling the study of in situ antigen localization under macrophage-free conditions.

\section{MATERIALS AND METHODS}

\section{Animals and chemicals}

Female BALB/c mice aged 12-16 weeks, were kept in macrolon cages under an $11 \mathrm{hr}$ dark $/ 13 \mathrm{hr}$ light regimen at $20^{\circ}$ and were given acidified water $(\mathrm{pH} 3)$ and pelleted mouse food (Hope Farms, Woerden, The Netherlands) ad libitum. DiI $\left(1,1^{\prime}\right.$ dioctadecyl-3,3,3',3'-tetramethylindocarbocyanine perchlorate; D-282) was obtained from Molecular Probes, Eugene, OR. Clodronate liposomes were prepared as described earlier. ${ }^{7}$

\section{Antigens}

Rabies virus (RV-Pasteur strain) was propagated in Vero-cell monolayer cultures. ${ }^{8}$ Culture supernatant was cleared by filtration and concentrated by Amicon ultrafiltration (cutoff $10^{6} \mathrm{MW}$ ). Virus was inactivated with $\beta$-propiolacton (BPL) and stored at $-70^{\circ}$ at a concentration of $900 \mu \mathrm{g} / \mathrm{ml}$. This rabies virus antigen was used for the preparation of RViscoms, as described earlier. ${ }^{9}$ Briefly RV-proteins were solubilized with $10 \%$ MEGA-10 (decanoyl- $N$-methylglucamide) for $2 \mathrm{hr}$. at room temperature. The solubilized virus was layered on top of a $10 \%$ sucrose cushion and centrifuged for $2 \mathrm{hr}$ at 200000 g. Quil-A (Iscotec AB, Luleå, Sweden), cholesterol and phosphatidylcholine (Sigma-Aldrich, Zwijndrecht, the Netherlands) were added to the supernatant and the mixture was dialysed overnight against phosphate-buffered saline (PBS). Free Quil-A and unincorporated proteins were removed by centrifugation over a $20 \%$ sucrose cushion for $48 \mathrm{hr}$ at $150000 \mathrm{~g}$. Incorporation of RV proteins was verified by sodium dodecyl sulphate-polyacrylamide gel eletrophoresis (SDSPAGE) analysis and a glycoprotein specific enzyme-linked immunosorbent assay (ELISA). ${ }^{10}$

\section{Immunological responses}

Clodronate liposomes were prepared as described in detail before, ${ }^{7}$ briefly: $75 \mathrm{mg}$ phosphatidylcholine and $11 \mathrm{mg}$ cholesterol were dissolved in chloroform in a $500 \mathrm{ml}$ round-bottomed flask. The thin film that formed on the interior of the flask after low vacuum rotary evaporation at $37^{\circ}$ was dispersed by gentle rotation for $15 \mathrm{~min}$ with $10 \mathrm{ml}$ PBS $(0.15 \mathrm{M} \mathrm{NaCl} / 10 \mathrm{~mm}$ phosphate buffer $\mathrm{pH} 7.4)$ containing $1.89 \mathrm{~g}$ dichloromethylene diphosphonate (a kind gift of Boehringer Mannheim, Germany). The milky white suspension was kept for $2 \mathrm{hr}$ at room temperature and sonicated for $3 \mathrm{~min}$ at $20^{\circ}$ in a Sonicor waterbath sonicator $(50 \mathrm{~Hz}$, Farmingdale, NY). After an additional $2 \mathrm{hr}$ at room temperature, the liposome suspension was diluted to $100 \mathrm{ml}$ in PBS and centrifuged $(100000 \mathrm{~g}$, $30 \mathrm{~min}$ ) to remove free clodronate. Liposomes were resuspended in $4 \mathrm{ml}$ PBS and microscopically checked for formation and size homogeneity. Three animals per group were injected i.v. with $200 \mu$ l Clodronate-liposomes or PBS intravenously. $48 \mathrm{hr}$ after treatment animals were immunized with $1 \mu \mathrm{g} \mathrm{RV}$ iscom or with $10 \mu \mathrm{g} \mathrm{RV-BPL}$. At day 40 animals were again treated with either Clodronate liposomes or with PBS and animals were booster immunized at day 42 . At day 49 animals were killed and the spleen was removed for T-cell proliferation studies. Immunological responses were measured 1 week after the last i.v. immunization at day 42. Rabies virus specific antibody responses were measured by antigen binding ELISA as described earlier. and T-cell responses were measured as described earlier. ${ }^{11}$ Briefly, spleen cells were isolated and stimulated with varying concentrations of RV-BPL. After 5 days cells were pulsed with $\left[{ }^{3} \mathrm{H}\right]$ thymidine for $18 \mathrm{hr}$. Cells were harvested on glass filters and incorporated radioactivity was measured in a Betaplate scintillation counter (LKB, Uppsala, Sweden). Sera were taken at weekly intervals and were analysed in a rabies-virus-specific ELISA for the induction of specific antibodies.

\section{Fluorochrome labelling of rabies virus antigen and $R V$-iscoms} Stock solution of DiI and was made in pure ethanol $(2.5 \mathrm{mg} / \mathrm{ml}) .100 \mu \mathrm{g} R$-BPL, RV-iscoms or empty iscoms were mixed with $20 \mathrm{mg}$ DiI in PBS and incubated for one hour at $37^{\circ}$. After labelling the samples were filtered using $0.45 \mathrm{~mm}$ filters to separate crystalline DiI and labelled antigens. ${ }^{12}$ Labelled material was stored in the dark at $4^{\circ}$ for up to 4 weeks. Administration was performed i.v. for several doses ranging from $0 \cdot 1$ to $10 \mu \mathrm{g}$ per mouse.

\section{Fluorescence microscopy}

Frozen sections $(8 \mu \mathrm{m})$ of organs under study were observed and photographed directly after cryo-sectioning with an Olympus (Tokyo) Vanox fluorescent microscope. DiI fluorescence was observed both as red with green light (excitation filter BP 545) and 'rhodamine/TRITC' optics (emission: EO 570).

\section{Enzyme cytochemistry}

Organ sections used for localization studies based on fluorescence microscopy were air-dried and fixed in acetone for enzyme staining. Endogenous acid phosphatase staining for the identification of all splenic and liver phagocytes was performed as described in detail earlier. ${ }^{13}$

\section{RESULTS}

\section{Localization of virus and iscom in the spleen after macrophage depletion.}

Already at 30 min after i.v. administration of DiI labeled RVBPL preferential uptake by marginal zone macrophages (MZM) could be observed in control mice (PBS at $-48 \mathrm{hr}$ ) with some antigen also being taken up by red pulp macrophages. This image was identical to the localization patterns we described in a recent study for normal untreated mice. ${ }^{3}$ The preferential uptake by MZM was maintained when observing sections at 60 or $120 \mathrm{~min}$ (Fig. 1a) and proved 

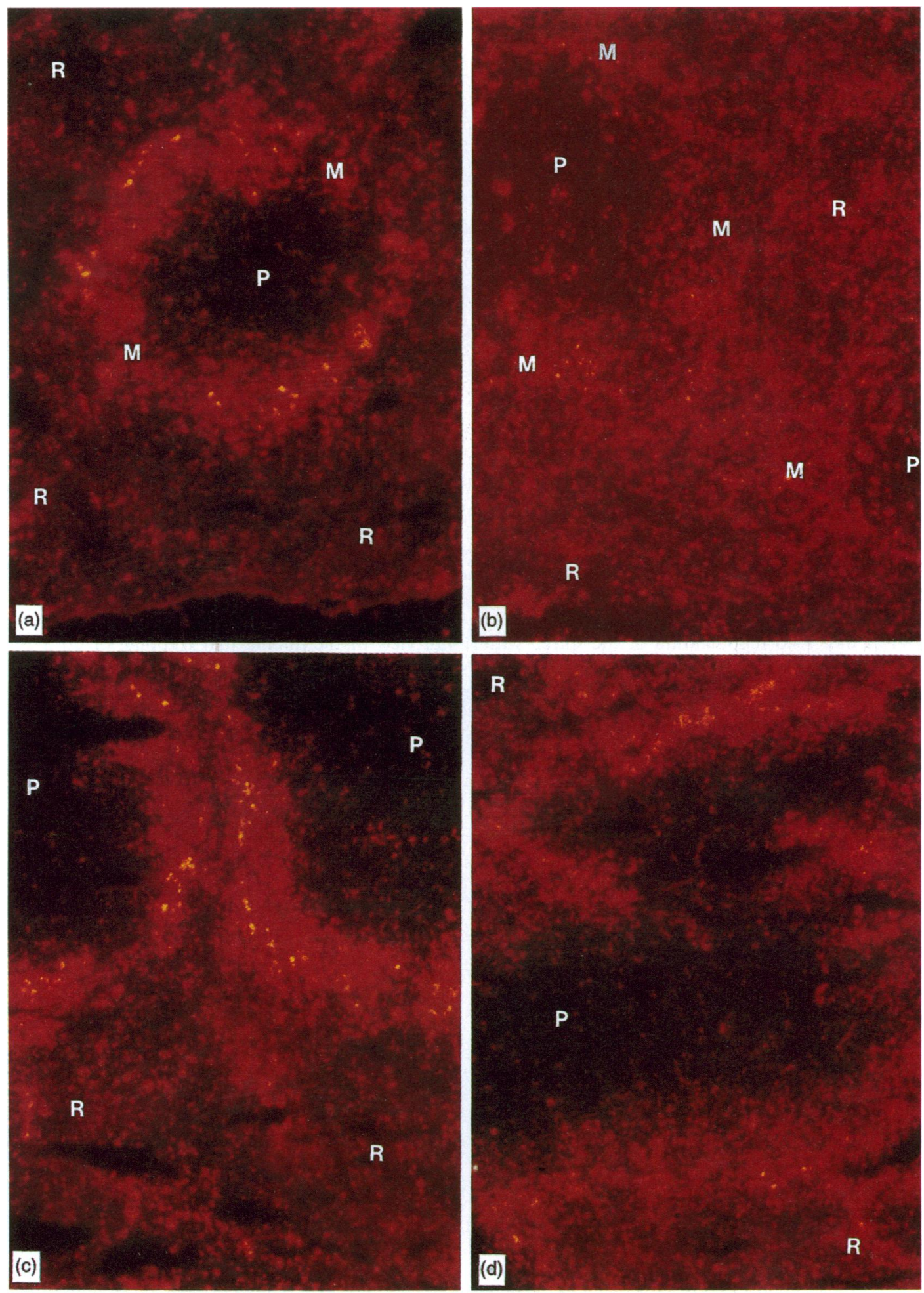

Figure 1. In this figure the uptake of DiI-labelled antigens is shown in the spleen $2 \mathrm{hr}$ after i.v. injection. (a) Uptake of RV-BPL in normal untreated animals. (b) Uptake of RV-BPL in clodronate treated animals. (c) Uptake of RV-iscom in untreated animals. (d) Uptake of RV-iscom in clodronate treated animals, R, red pulp, M, marginal zone, P, peri-arteriolar lymphocyte sheath.

completely in accordance with other localization studies on particulate thymus dependent antigens. ${ }^{14,15}$

Splenic macrophages were depleted by i.v. administration of clodronate liposomes, ${ }^{16}$ depletion was complete as confirmed with enzyme histochemistry for acid phosphatase of alternating sections from those shown in Fig. 1 (data not shown). Irrespective of this complete absence of macrophages from the spleen, a massive trapping and retention of DiIlabelled RV-BPL could be observed (irrespective of the dose given). Contrary to the discrete localization pattern found in 
normal or PBS treated mice (in MZM and red pulp), RV-BPL seemed to be evenly distributed over all splenic compartments (Fig. 1b).

As described before, ${ }^{3}$ iscom was taken up preferentially by MMM (bright yellow dots in Fig. 1c) and to a lesser extent by MZM and on cells in the follicles. In macrophage-depleted animals iscom was trapped and retained almost exclusively in the marginal zone and red pulp of the spleen (irrespective of the dose given). Contrary to RV-BPL almost no iscom localization could be observed in the PALS area of depleted animals (Fig. 1d).

\section{Immunological responses after depletion of spleen macrophages}

Based on dose-response curves generated before ${ }^{3}$ we chose for similarity of eventual titre rather than dose of immunization (antigen). In this scenario $1 \mu \mathrm{g}$ of $\mathrm{RV}$-iscom is equivalent to $10 \mu \mathrm{g}$ of RV-BPL, both resulting in virus neutralising antibodies, specific $\mathrm{T}$ cells and $100 \%$ survival after challenge (i.e. full protection). ${ }^{3}$

Rabies-virus-specific antibody responses in serum were measured weekly in antigen-specific ELISA. Serum samples were analysed weekly after depletion of splenic macrophages and subsequent immunization to observe possible effects caused by macrophage repopulation of the spleen. It was established that at day 7 no repopulation of the spleen with macrophages has yet occured, marginal metallophils reappear between 14 and 21 days and at day 28 the normal situation is almost completely restored ( ${ }^{4,6,17}$ and data not shown). As is shown in Fig. 2(a) (represenative experiment from three performed) no difference in the kinetics of the antibody response could be observed when splenic macrophages were removed in $\mathrm{RV}$-iscom immunized animals as compared with untreated animals. It should be noted that a trend towards higher titers in depleted RV-BPL animals could be observed (not significant).

The clodronate treatment was repeated at day 40 and animals were booster immunized with RV-iscom at day 42 . Both treated and untreated animals showed increased serum titres at 7 days after boost immunization as compared to primary responses. Again, no effect of the clodronate liposome treatment was observed. Identically, humoral responses after immunization with RV-BPL were unaffected after clodronate liposome treatment.

Proliferative splenocyte responses in RV-BPL immunized animals were unaffected after treatment with clodronate liposomes (Fig. 2c). However, when splenic macrophages were removed prior to immunization with $\mathrm{RV}$-iscom a significant decrease in rabies-virus-specific spleen-cell responses could be observed (Fig. 2d). In vitro restimulation of T cells with either inactivated antigen or antigen as provided by paraformaldehyde (PFA)-fixed persistently ERA (Evelyn-RokitnickyAbelseth)-infected P815 (H2d, haplotype) cells was also significantly decreased in clodronate liposome-treated animals.

\section{DISCUSSION}

In this study we show that the T-cell mediated immune response against rabies virus iscom is partly dependent on splenic macrophages. Depletion of macrophages resulted in almost identical in situ localization patterns for rabies virus antigen presented as inactivated virus or in iscom. The elimination of splenic macrophages has no effect on the humoral response against rabies virus for both antigen formulations.

When given in sufficiently high doses, 'empty' liposomes can efficiently block and suppress the macrophage system. ${ }^{18,19}$ Similarly, macrophages can be suppressed or depleted by using substances such as carrageenan, silica, carbonyl iron or antibodies. ${ }^{20}$ We have developed and applied a liposome-suicide technique for the actual elimination of macrophages. ${ }^{7,16}$ The resulting modulation studies have firmly established the role of macrophages in innate (antigen removal) rather than in adaptive (antigen presentation) immunity., ${ }^{4,5}$ Emphasizing the role of what is now called professional antigen presenting cells, such as dendritic and B cells, in the generation of antibodies and cellular immunity. ${ }^{5}$ Not very surprising it could be established in a similar fashion that the macrophage is the principal antigen-presenting cell for liposome-encapsulated antigens $^{21}$ as well as for other particulate antigens. ${ }^{15,22}$ Furthermore, CTL responses induced by membranous vesicles but not those induced by syngeneic antigen pulsed splenocytes proved to be macrophage dependent ${ }^{23}$. Iscom and inactivated virus can be regarded as hydrophobic particulate antigens. In this study no differences in humoral response were observed in macrophage-depleted cells as compared with untreated animals, at the antigen dose and immunization regime studied. In view of the finding that trapping of both antigens occurs on follicular cells in depleted animals it can be concluded that follicular dendritic or follicular B cells are involved in antigen presentation of both RV-BPL and iscom. This observation, in combination with the observed decrease in T-cell responses against iscom in depleted animals, suggests a role for both follicular cells and MMM in the presentation of iscom. And no or very limited role for MMM in the presentation of RV-BPL.

To induce an efficient MHC class I restricted immune response, involving the production of antigen specific cytotoxic T-cells (CTL), it seems necessary to target the antigen to the cytoplasm ${ }^{29}$ or induce processing by dedicated macrophages $^{22-24}$. It was shown that both anionic and cationic ${ }^{32}$ pH-sensitive liposomes could enter the class-I restricted (endoplasmatic reticulum/Golgi) pathway. ${ }^{22,24,25}$ The addition of Quil A to liposomes has been shown to efficiently enhance their capacity to induce CD8 ${ }^{+} \mathrm{CTL} .{ }^{26}$ Quil-A is an adjuvant and toxic by itself, and a crucial component of iscom which belong to the most potent adjuvants, for hydrophobic proteins, known. ${ }^{1-3}$ Both rabies virus iscom and RV-BPL can induce antigen-specific, class-I restricted CTL. Quil A containing liposomes proved to be an effective vehicle to shuttle hydrophillic proteins into the major histocompatibility complex (MHC) class I pathway, resulting in induction of $\mathrm{CD}^{+}$cytotoxic T lymphocytes (CTL). These and other ${ }^{4,27}$ data point to a conflicting role for macrophages in direct stimulation of $T$ cells in some systems and suppression of $T$ cells in others.

We have shown previously that the T-cell responses are not directly influenced by treatment with clodronate liposomes. ${ }^{28}$ Consequently the observed decrease in T-cell proliferation in RV-iscom immunized animals is likely a result from the absence of splenic macrophages. Because no decrease was observed in the CD4 T-cell dependent humoral response the observed decrease can be explained by in the class-1 restricted 
(a)

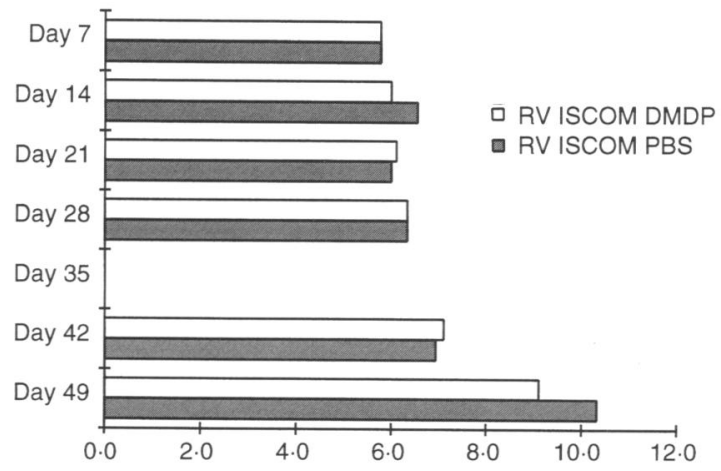

(b)

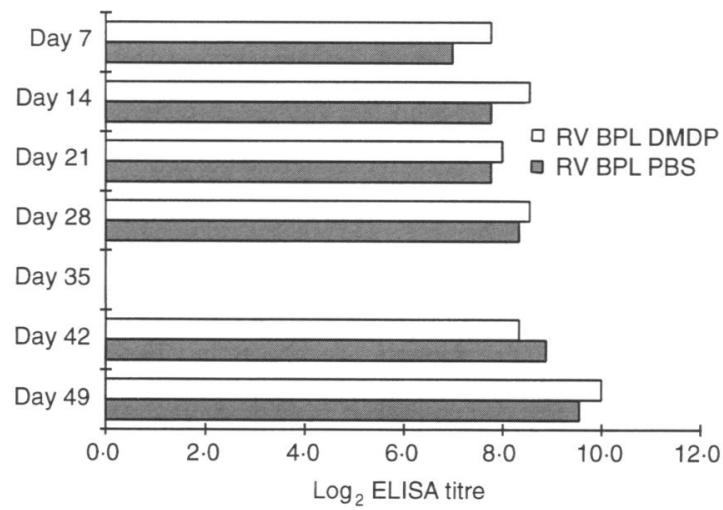

(c)

RV-BPL

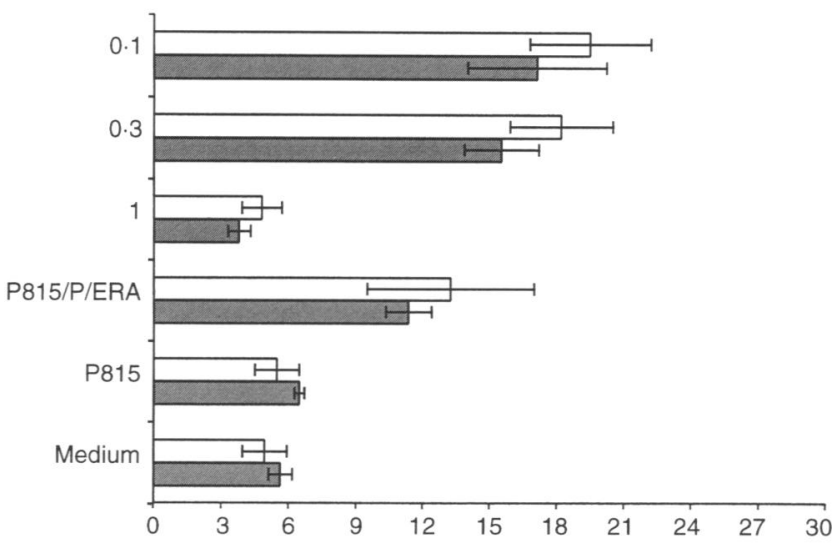

(d)

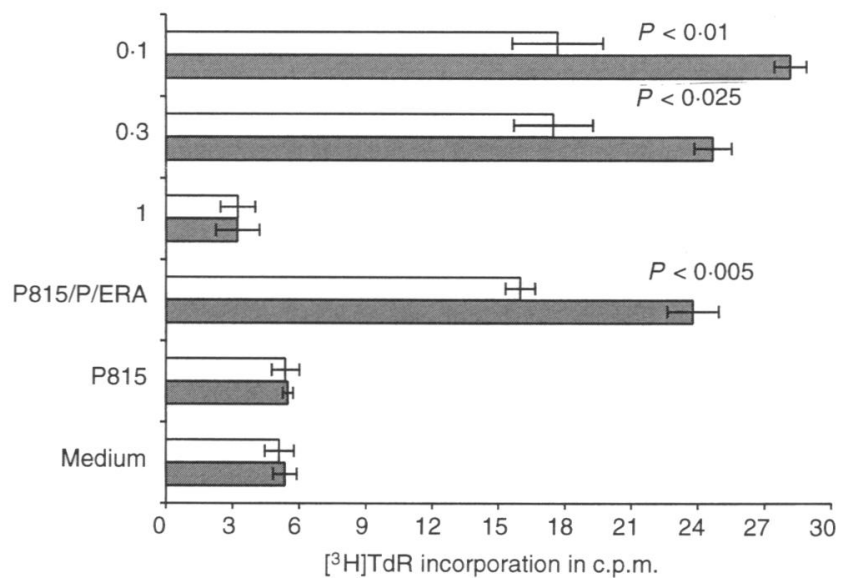

Figure 2. In this figure the rabies virus specific immunological responses are shown before and after treatment with clodronate liposomes. Rabies virus specific ELISA serum titres in RV-BPL (a) and RV-iscom (b) immunized animals immunization. Proliferative spleen cell responses in RV-BPL (c) and RV-iscom (d) immunized animals.

CTL response which is dependent on the presence of macrophages. Such a decrease has been described for the generation of primary ovalbumin (OVA)-specific CTL after splenic macrophage elimination. ${ }^{29}$ Why this decrease does not occur in RV-BPL immunized animals, which normally generate CTL activity as well, remains unclear but, in combination with the localization data, it suggests a role for the MMM subset in the (pre)processing of antigen in iscom and not in other unactivated antigen forms.

Removal of splenic and hepatic macrophages with clodronate liposomes is very efficient and actually eliminates phagocytic cells from the tissues altogether. ${ }^{16}$ As described before in studies using radioactively labelled liposomes ${ }^{30}$ this removal does not result in persistently increased serum levels of the antigen nor in a decrease of splenic uptake or a change of antigen localization patterns. The present results show the localization patterns of this nonphagocytic trapping by the spleen. As observed for a soluble thymus-independent antigen ${ }^{5}$ it is evident that antigen is still trapped by follicular (dendritic or B) cells of the spleen after macrophage depletion. In view of the crucial role of dendritic cells ${ }^{29}$ and follicular dendritic cells in the presentation of antigens to the immune system ${ }^{3,5}$ this is an important observation. Furthermore, this is in accordance with the primary function of the spleen, namely as a filter of the blood. ${ }^{4,5}$ In a large number of studies it has now been shown that removal of marginal zone macrophages leads to a relative increase of humoral immune responses against particulate antigens ${ }^{17}$ or antigens in oil emulsions. ${ }^{31}$ This trend is also visible in this study (Fig. 2b). The preferential localization of iscom in MMM certainly leads to relative protection of the antigen for the degradable action of marginal zone macrophages and provides an explanation for the relative effectiveness (10-25-fold efficiency gain in terms of dose $^{3}$ ) of iscom in inducing immune responses. Studies involving development or optimization of iscom or other antigen presenting moieties should therefore include similar in vivo localization and modulation studies to better predict eventual efficacy. This in turn will lead to vaccines with higher efficacy at much lower antigen concentrations because much less antigen is lost by immunologically non-specific filtering.

\section{REFERENCES}

1. Morein B., Sundquist B., Hoglund S., Dalsgaard K. \& Osterhaus A. (1984) Iscom, a novel structure for antigenic presentation of membrane proteins from enveloped viruses. Nature 308, 457. 
2. Claassen I.J.T.M. \& Osterhaus A.D.M.E. (1992) The iscom structure as an immune enhancing moiety: experience with viral systems. Res Immunol 143, 531.

3. Claassen I.J.T.M., Osterhaus A.D.M.E. \& Claassen E. (1995) Antigen detection in vivo after immunization with different presentation forms of rabies virus antigen: involvement of marginal metallophilic macrophages in the uptake of immune stimulating complexes. Eur J Immunol 25, 1146.

4. Laman J.D. \& ClaAassen E. (1995) T cell independent and T cell dependent humoral immunity. In: Cytokine Regulation of Immunoglobulin Synthesis and Class Switching (ed. C. Snapper), p. 23. John Wiley \& Sons, New York.

5. Van den Eertwegh A.J.M., Laman J.D., Schellekens M.M., Boersma W.J.A., ClaAssen E. (1992) Complement mediated follicular localization of T-independent antigens: the role of marginal zone macrophages revisited. Eur J Immunol 22, 719.

6. Delemarre F.G.A., Kors N., Kraal G. \& Van Rooijen N. (1990) Repopulation of macrophages in popliteal lymph nodes of mice after liposome treated depletion. $J$ Leukocyte Biol 47, 251.

7. Claassen E. \& van Rooijen N. (1986) Preparation and characteristics of dichloromethylene disphosphonate-containing liposomes. J Microencapsulation 3, 109.

8. van Wezel A.L., van Steenis B., Hannik C.A. \& Cohen H. (1978). New approaches to the production of concentrated and purified inactivated polio and rabies tissue culture vaccines. Dev Biol Standard 41, 159.

9. Fekadu M., Shaddock J.H., Ekstrom J. et al. (1992) An immune stimulating complex (ISCOM) subunit rabies vaccine protects mice and dogs against street rabies challenge. Vaccine 10, 192.

10. Thraenhart O., Koprowski H., Bögel K. \& Sureau P. (eds) (1990) Progress in Rabies Control. Staples Printers, Rochester Ltd, p. 69.

11. Bunschoten H., Dietzschold B., ClaAssen I.J.T.M., Klapmuts R., UytdehaAg F.G.C.M. \& Osterhaus A.D.M.E. (1990) Rabies virus cross-reactive murine $\mathrm{T}$ cell clones: analysis of helper and delayed-type hypersensitivity function. Viral Immunol 3, 41.

12. Claassen I., Osterhaus A., Boersma W., Schellekens M. \& ClaAsSEN E. (1995) Fluorescent labelling of virus, bacteria and iscoms: in vivo systemic and mucosal localisation patterns. In: Advances in Mucosal Immunology (eds P. Brandtzaeg, J. McGhee, J. Mestecky, J. Sterzl \& M. Tlaskalova) p. 1485 Plenum Press, New York.

13. Claassen E., Westerhof Y., Versluis B., Kors N., Schellekens M. \& VAN RooIJEN N. (1988) Effects of chronic injection of sphingomyelin containing liposomes on lymphoid and nonlymphoid cells in the spleen. Transient suppression of marginal zone macrophages. Br J Exp Pathol 69, 865.

14. Buiting A.M.J., De Rover Z., ClaAssen E. \& van Rooijen N. (1993) In vivo distribution of particulate antigens and liposomes in murine spleen. A possible role in the humoral immune response. Immunobiology 188, 13.

15. VAN RooIJEN N. (1992) Macrophages as accesory cells in the in vivo humoral response: from processing of particulate antigens to regulation by suppression. Sem Immunol 4, 237.

16. VAN Rooijen N. \& SANDERS A. (1994) Liposome mediated depletion of macrophages: mechanism of action, preparation of liposomes and applications. J Immunol Methods 174, 83.
17. Claassen E. (1996) Liposomes in in vivo Immunology. In: Vesicles (ed. M. Rosoff), Surfactant Science Series, Vol. 62, p. 649. Marcel Dekker Inc., New York.

18. Juliano R.L. (1982) Liposomes and the reticuloendothelial system: interactions of liposomes with macrophages and behaviour of liposomes in vivo. In: Targeting of Drugs (eds, G. Gregoriadis, J. Senior \& A. Trouet), NATO ASI Series A, Vol. 47, p. 285. Plenum Press, New York.

19. Proffitt R.T., Williams L.E., Presant C.A. et al. (1983) Liposomal blockade of the reticuloendothelial system: improved tumor imaging with small unilamellar vesicles. Science 220, 502.

20. Leblanc P.A. \& Russell S.W. (1981) Depletion of monnuclear phagocytes: Pitfalls in the use of carbonyl iron, carrageenan, silica, trypan blue, or antimononuclear serum. In: Methods for Studying Mononuclear Phagocytes (eds D. O. Adams, P. J. Edelson and H. S. Koren), p. 231. Academic Press, New York.

21. SzoKA F.C. JR. (1992) The macrophage as the principle antigen presenting cell for liposome encapsulated antigen. Res Immunol 143, 186.

22. VAN Rooljen N. (1995) Liposome mediated immunopotentiation and immunomodulation. In: Vaccines: New-Generation Immunological Adjuvants (ed. G. Gregoriadis), p.15. Plenum Press, New York,

23. ZhOu F., Rouse B.T. \& HuANG L. (1992) Induction of cytotoxic $\mathrm{T}$ lymphocytes in vivo with protein antigen entrapped in membranous vesicles. $J$ Immunol 149, 1599.

24. Zhou F. \& HuAng L. (1994). Liposome-mediated cytoplasmic delivery of proteins: an effective means of accessing the MHC class I-restricted antigen presentation pathway. Immunomethods 4, 229.

25. Martin S., Niedermann G., Leipner C. \& Eichmann K.\&. (1993) Weltzien.Intracellular processing of hapten-modified protein for MHC class I presentation: cytoplasmic delivery by $\mathrm{pH}$-sensitive liposomes. Immunol Lett 37, 97.

26. Piera M., de Bolos C., Castro R. \& Real F.X. (1993) Cytokines as adjuvants: effect on the immunogenicity of NeuAc alpha 2-6 GalNac alpha-O-Ser/Thr (sialyl-Tn). Int J Cancer 55, 148.

27. Croft M. (1994) Activation of naive, memory and effector $T$ cells. Curr Opin Immunol 6, 431.

28. Claassen I., van Rooijen N. \& Claassen E. (1990) A new method for removal of mononuclear phagocytes from heterogenous cell populations in vitro, using the liposome-mediated macrophage 'suicide' technique. J Immunol Methods 134, 153.

29. Nair S., Buiting A.M.J., Rouse R., van Rooijen N., Huang L. \& Rouse B.T. (1995) Role of macrophages and dendritic cells in primary cytotoxic T lymphocyte responses. Int Immunol 7, 679.

30. Claassen E. \& van Rooijen N. (1984) The effect of elimination of macrophages on the tissue distribution of liposomes containing 3 (H) methotrexate. Biochem Biophys Acta 802, 428.

31. Leenaars P.P.A.M., Hendriksen C.F.M., Savelkoul H.F.J., VAN RooIJEN N. \& ClaAsSEN E. (1997) Increased adjuvant efficaccy in stimulation of antibody responses after macrophage elimination. Immunology 90, 337.

32. Wizel B., Rogers W.O., Houghten R.A., Lanar D.E., Tine J.A. \& HofFman S.L. (1994) Induction of murine cytotoxic T lymphocytes against Plasmodium falciparum sporozoite surface protein 2. Eur J Immunol 24, 1487. 